\title{
Acción, historia y naturaleza en el pensamiento de Hannah Arendt
}

\begin{tabular}{l} 
Action, history and nature \\
of thought Hannah Arendt \\
\hline
\end{tabular}

Ação, historia e natureza no pensamento de Hannah Arendt

Fecha de entrega: 24 de agosto de 2013 Fecha de evaluación: 8 de noviembre de 2013 Fecha de aprobación: 20 de diciembre de 2013

Gloria Gallo*

\section{Resumen}

El objetivo de este trabajo es plantear la relación que establece Hannah Arendt entre los conceptos de acción, historia y naturaleza, cuya conexión se da en torno a las características que hacen a las acciones humanas contingentes, es decir, la imposibilidad de predecir sus consecuencias y sus efectos ilimitados. El análisis de las relaciones entre los conceptos aquí aludidos es una herramienta de reflexión sobre la necesidad de replantear la relación entre el hombre y la naturaleza. Inicialmente, haré una breve exposición de la tesis de la acción y la vida política del hombre. Seguidamente, expondré los aspectos por los cuales la historia y la naturaleza pueden ser comprendidas en

Licenciada en Filosofía y Letras de la Universidad Santo Tomás, magister en Filosofía de la Universidad el Rosario. Profesora de la Escuela de Ciencias Humanas de la Universidad del Rosario, Bogotá. Realiza investigaciones sobre filosofía política contemporánea. Correo electrónico: gloria.gallo@gmail.com 
correspondencia a la categoría de la acción política, para finalmente exponer algunas reflexiones que pueden surgir de esta interpretación en torno a los efectos impredecibles de la intervención humana sobre la Tierra.

Palabras clave: labor, trabajo, acción, política, historia, naturaleza, ciencia, técnica.

\section{Abstract}

The purpose of the present study is to expose the relationship Hannah Arendt establish among the action, history and nature's concepts, of which connection is given around the characteristics that make human actions to be contingents, which means, the impossibility to predict their own consequences and limited effects.. The analysis of the relationship among the concepts here exposed, could be a reflection tool about the necessity of rethinking the relationship between man and nature. A brief exposition of the thesis of the action and man's political life will be made. Then, the aspects for which the history and nature could be comprehend as a correspondence of the political action category will be comment, to finally exposure some of the reflections that could follow this interpretation around the unpredictable effects of human's intervention on earth.

Keywords: Labor, work, action, politics, history, nature, science, technique. 


\section{Resumo}

O objetivo deste trabalho é plantear a relação que estabelece Hannah Arendt entre os conceitos de ação, história e natureza, cuja conexão é dada em torno às características que fazem às ações humanas contingentes, quer dizer, a impossibilidade de predizer suas consequências e seus efeitos ilimitados. A análise das relações entre os conceitos falados aqui é uma ferramenta de reflexão sobre a necessidade de reconsiderar a relação entre o homem e a natureza. No início, farei uma simples exposição da tese da ação e a vida política do homem. A seguir, vou expor as razões pelas quais a história e a natureza podem ser compreendidas em concordância à categoria da ação política, para no final, expor algumas reflexões que podem surgir desta interpretação ao redor dos efeitos imprevisíveis da intervenção humana sobre a Terra.

Palavras-chave: labor, trabalho, ação, política, história, natureza, ciência, técnica. 
En La condición Humana, Arendt plantea una relación entre las consecuencias de la manipulación de la naturaleza y los logros de la ciencia contemporánea con aquellas características propias de la acción humana. Para comprender este punto, es importante plantear brevemente algunos aspectos generales sobre su tesis de la acción.

En este libro, Arendt diferencia tres actividades que pueden identificarse como condiciones de la vida de los hombres; estas son las siguientes. Primero, está la labor, la cual corresponde al proceso biológico y se relaciona con todas las actividades que los hombres realizan para superar las necesidades vitales. Segundo, se encuentra el trabajo, que es un proceso no biológico por el cual los hombres fabrican objetos, o bien para configurar un mundo con ellos o bien para ponerlos en el mercado como objetos de consumo. Finalmente, Arendt identifica la acción como una actividad que se da sin la mediación de cosas, puesto que se da entre los hombres y su condición es la pluralidad cuyo objetivo es crear cuerpos políticos. El modelo al que recurre Arendt, para describir lo que fue y lo que podría plantearse como el deber ser de lo político, es el griego.

Considero importante resaltar que la distinción hecha por Arendt entre la labor, el trabajo y la acción tiene como finalidad establecer que solo la acción posibilita a los hombres reconocerse y ser reconocidos como diferentes, y este es el criterio más destacado para la pluralidad como elemento fundante y permanente de toda comunidad política. Frente al laborar y al fabricar (actividades que se acometen con el fin de solucionar las necesidades inmediatas y producir los objetos del mundo en el que los hombres se interrelacionan) se encuentra la acción, esa actividad por medio de la cual los hombres se dirigen unos a otros, y que unida al discurso, posibilita el hecho que estos se revelen entre sí. Es decir, se reconozcan mutuamente como un otro distinto. En La condición humana, Arendt afirma que, aunque todas las actividades humanas tienen como atributo el hecho de que los hombres pueden realizarlas en la cercanía de unos con otros, solo la acción es inimaginable sin la presencia de los demás. Esta mutua presencia en las acciones humanas es la que da origen a la comunidad política, no a la sociedad, conceptos que Arendt diferencia con el fin de explicitar las razones por las cuales se ha confundido lo público (político) con lo privado (social).

En la tradición, desde la Edad Media hasta la Modernidad, el carácter político del hombre y de la acción se confundió con el carácter social, diferencia que, según Arendt, los griegos tenían muy clara. De esta manera, la condición social del hombre 
está determinada por el ámbito privado, es decir por las relaciones familiares de una persona y las que tiene con sus amigos; mientras que el carácter político de los hombres está determinado por las relaciones públicas entre ellos y su deseo de unirse para conformar una comunidad política. En esta visión, el carácter social del hombre es igual al de los animales, pues es la necesidad la que genera la urgencia de agruparse en familias. En la interpretación política de Arendt, los griegos excluyeron los otros modos de vida sociales o asociaciones naturales cuyo centro es el hogar (oikia). La fundación de la polis se dio por fuera de otros tipos de organización basados en el parentesco (phratria y phylie).

Es de resaltar que esta crítica sobre el ser político que presenta de Arendt es radical y pone al hombre del mundo contemporáneo en el nivel de los animales, que están asociados únicamente con la finalidad de la supervivencia. Según ella, lo que sí es propio de los hombres es su capacidad para salir a la esfera pública (pero tal capacidad se encuentra actualmente en crisis), pues esta los separa y distingue de los otros animales. Tal carácter distintivo está determinado por el hecho de que la forma en que los hombres entran en la esfera pública es a través de la acción y la palabra, y así lo entendieron los griegos. Las actividades humanas propias del bios politikos son la acción (praxis) y el discurso (lexis).

Tal y como ocurre con su tesis sobre la acción política, Arendt recurre a la experiencia griega para plantear el sentido de la historia; no obstante, su interés no radica en hallar la definición del concepto mismo sino en plantear cuál será su tarea. De tal forma, Arendt recuerda que Herodoto:

\begin{abstract}
En la primera fase de su obra sobre las Guerras Persas nos dice que el objetivo de su esfuerzo es preservar lo que nació por obra de los hombres (Ta Yevoyeva eg ávdpwnwv), para que el tiempo no lo borrara y para otorgar a las hazañas gloriosas, admirables de los griegos y los bárbaros la alabanza suficiente que asegurase que la posteridad habría de recordarlas y así mantendría impoluta esa gloria a través de los siglos. (Arendt, 1996a, p. 49)
\end{abstract}

De allí Arendt concluye que Herodoto, como representante del espíritu griego, tiene claro que la tarea de la historia es "salvar las hazañas humanas de la trivialidad que se deriva del olvido" (Arendt, 1996a, p. 49). 
De esta concepción hay varios aspectos para resaltar. Por una parte, se puede enfatizar el énfasis en lo individual, por la historia de cada hombre en particular; esta individualidad está sometida a la mortalidad frente a la inmortalidad de la naturaleza. Por otro lado, se puede destacar la relación de este énfasis en lo individual con la tesis de la acción. Finalmente, se puede acentuar la pretensión del hombre moderno de hacerse a la infinitud a través de una concepción del mundo, de la historia y de sí mismo, sustentada en los procesos colectivos, lo cual suprime el sujeto en su carácter particular y temporal.

Respecto al primer aspecto, es necesario aclarar cuál es la relación entre los hombres y la naturaleza. Mientras a la naturaleza le es inmanente la eternidad a través de la repetición del ciclo eterno de la vida, al hombre como individuo (aunque como especie haga parte de ese mismo ciclo eterno) le es dada la mortalidad. Existen además elementos que son producidos solamente por los hombres; estos son las palabras y las acciones y también son perecederos.

Para Arendt, la tarea de la historia, tal y como fue concebida por los griegos es hacer memorables las acciones, las palabras y acontecimientos que merecen serlo. La historia es la de un héroe, es individual y tiene como finalidad inmortalizar aquello que es por naturaleza mortal. Si las acciones, las palabras y los acontecimientos son realizados por hombres particulares quienes se caracterizan por su mortalidad, entonces hay una paradoja frente a la naturaleza que es inmortal, frente a los ciclos de la vida que son eternos. Esta paradoja fue solucionada por los griegos mediante la historiografía y la poesía.

En la concepción griega de la historia el individuo ocupa un lugar predominante, el hombre y sus acciones, sus palabras y acontecimientos son el tema de la historia tal y como la entendieron los griegos. En esta visión los hombres que son dignos de ser recordados intentan alcanzar mediante la historia la inmortalidad que solo le es propia a la naturaleza. Existe pues una relación entre la historia y la naturaleza: según Arendt, "su común denominador es la inmortalidad" (1996a, p. 56).

En oposición a esta visión, la actitud del pensamiento moderno frente a la naturaleza estuvo impulsada por su deseo de suprimir la subjetividad, el punto de vista del observador, actitud que de alguna manera fue imitada por la historia moderna. Para Arendt, en la modernidad también existe una relación estrecha entre el concepto de historia y naturaleza. La objetividad con que el investigador intentaba acercarse al 
conocimiento de la naturaleza implicaba eliminar el punto de vista del observador, como si la naturaleza misma pudiera revelar sus leyes sin la necesidad de que el investigador realizara pregunta alguna. La historia pretendía tomar el mismo camino. La objetividad en este caso se lograba de dos maneras. En primer lugar, está la no discriminación, lo cual implica que el historiador elimina su propio punto de vista y relate solamente el curso de los acontecimientos tal y como se lo revelan las fuentes. En segundo lugar, se encuentra la no interferencia según la cual el investigador trata de no injerir en las condiciones que el historiador desea revelar. Para Arendt, esta última condición es imposible de alcanzar, puesto que cualquier selección de material implica una interferencia por parte del historiador, porque pone los hechos bajo las condiciones de selección que ha realizado.

Sin embargo, Arendt observa que esta actitud ha cambiado en el contexto de la ciencia contemporánea. Como testigo de los avances en la física nuclear y de la puesta en el espacio de los primeros satélites, resalta que el observador se convierte en una más de las condiciones que va a determinar la experimentación científica. Algo así como si en la investigación, las ciencias de la naturaleza agregaran un nuevo elemento subjetivo.

El actual problema es que las ciencias contemporáneas han asumido una actitud nueva con respecto a los fenómenos que investigan, con la que el punto de vista del investigador ha entrado a formar parte de la indagación sin que por ello la ciencia pierda su rigor. Empero, "[e]l punto curioso y todavía confuso en las ciencias históricas es que no adoptaron las normas de las ciencias naturales de su misma época, sino que se volvieron hacia la actitud científica y, en última instancia filosófica que la época moderna había empezado a liquidar" (Arendt, 1996a, p. 58). La historia se empeñó en imitar la actitud moderna de las ciencias en búsqueda de la objetividad, pero en el presente se encuentra incapaz de imitar nuevamente a las ciencias de la naturaleza y, por tal razón, Arendt encuentra que se ha rezagado frente al avance de las ciencias naturales.

El rasgo característico de la historia, como fue legada por Homero, Herodoto y Tucídides, radica en la imparcialidad; esto significa que los acontecimientos o hazañas narradas son tan grandiosas por sí mismas que quien las relata solo debe cumplir con la tarea de conservar su grandeza y transmitir su gloria. Hay aquí un énfasis en las hazañas y acontecimientos individuales que por su fama no necesitan más que ser contados para que permanezcan. De esto también se infiere que si la historia busca 
la inmortalidad lo hace despreciando la vida efímera del hombre que contrasta con la eternidad de la naturaleza. Esta era, según Arendt, la imparcialidad clásica de la historiografía.

En el pensamiento moderno, la imparcialidad es entendida como objetividad, la cual no es para nada comparable con la forma en que los griegos comprendieron la imparcialidad de la historia, para ellos esta era validada por la experiencia individual de quien realizaba las hazañas dignas de contarse. Mientras que, para los griegos, la historia significaba enaltecer hechos y hazañas singulares, la objetividad moderna rechazó de plano la subjetividad, lo singular.

El deseo de objetividad en las ciencias naturales planteó un conflicto, que hasta hoy no se encuentra resuelto, sobre si el conocimiento de la verdad de la naturaleza se alcanza o bien por revelación de las leyes mismas que la rigen o bien por la desconfianza en lo que los sentidos muestran al hombre. La aún insoluble disputa entre racionalistas y empiristas sobre qué podemos conocer ha pasado a un segundo plano. Arendt afirma que el interés se ha desplazado hacia cómo conocemos, de ahí el interés por los procesos, entendiendo proceso como aquello que el hombre hace o inicia, es decir, todo aquello que ocurre por la acción humana. No obstante, a pesar de que las acciones humanas son impredecibles, el pensamiento moderno cree firmemente, desde la noción de proceso, que todo aquello que inicia tiene un principio y, por ende, un fin que él mismo puede controlar o prever.

Arendt llega a la noción de proceso a través del pensamiento de Giambattista Vico, quien, a pesar de ser contemporáneo de Descartes, no se sintió influenciado por la fama de su pensamiento sino que, por el contrario, planteó la imposibilidad del conocimiento de la naturaleza debido a que, según él, los hombres solo pueden conocer lo que hacen. Desde este punto de vista, lo único de lo que los hombres pueden tener pleno conocimiento es de la historia puesto que es la única cosa que los hombres hacen. Sin embargo, según Arendt, es hoy la técnica la que hace lo que Vico creía que hacía la historia: esta inicia procesos, los cuales surgen a partir de la manipulación de los elementos mismos de la naturaleza, mientras que la historia actual (como ciencia) no es capaz de proceder de esta manera, pues esta tarea le ha sido legada, según la autora, a las ciencias sociales.

El inconveniente radica en que a pesar de que los hombres creen controlar estos procesos que ellos mismos producen, esto no ocurre en la realidad. Si la acción humana 
se caracteriza por ser impredecible e ilimitada, igual sucede con los procesos que el hombre desencadena, los que son fruto de las ciencias sociales y los que son fruto de la tecnología. Con respecto a los primeros, es bien conocida la actitud crítica que tiene Arendt frente a las ciencias sociales, actitud que, para algunos, como Giorgo Agamben, es precursora de la postura de Foucault frente a estas mismas ciencias que han aparecido recientemente en la historia del pensamiento. De acuerdo con esta pensadora:

Las ciencias sociales, comparativamente nuevas, que con tanta rapidez pasaron a ser a la historia lo que la tecnología resultó ser a la física, pueden usar la experimentación de un modo mucho más tosco y menos fidedigno que las ciencias naturales, pero el método es el mismo: también prescriben condiciones, condiciones para el comportamiento humano, así como la física prescribe condiciones para los procesos naturales. Si [...] nuestra deplorada impotencia para 'gestionar' los asuntos humanos, a través de una ciencia de ingeniería de las relaciones humanas, suena aterradora, es solo porque han decidido tratar al hombre como un ser absolutamente natural cuyo proceso de vida puede manipularse del mismo modo que los demás procesos. (Arendt, 1996a, p. 68)

Por otra parte, el problema con los procesos desencadenados por la tecnología, es que han desatado fuerzas que amenazan con destruir todo lo que existe en el planeta. En el contexto histórico de Arendt, esto se refería a la competencia armamentista nuclear de la Guerra Fría; en el contexto contemporáneo, es el desequilibrio ocasionado por la incesante competencia económica y, por ende, el uso indiscriminado de fuentes de energía altamente competitivas, pero también altamente contaminantes.

Este tratamiento sobre la naturaleza, propio de la modernidad y del mundo contemporáneo, tiene su origen en lo que Arendt llama el triunfo del homo faber. El hombre creyó -tanto a través del desarrollo de la ciencia como del auge de la mecanizaciónque la naturaleza se encontraba dispuesta para su uso como materia en la fabricación del artificio humano. El mundo natural se vio entonces sujeto al sentido que solo le es dado a los objetos que el hombre fabrica. Esto generó la pérdida del espacio político, y se impuso, así, el mundo de la necesidad, pues los hombres solo se interrelacionan entre sí y con el mundo, de acuerdo con sus intereses económicos que antes estaban supeditados al ámbito privado. 
Esta actitud del hombre frente a la naturaleza, guiada por los intereses vitales, no simplifica al hombre, devolviéndolo al nivel de los animales por la pérdida de ese espacio político de aparición, también ha trasladado el carácter impredecible de la acción al artificio humano. Esta situación es aún más peligrosa en el contexto de la ciencia contemporánea porque lo que el hombre ha generado a partir de los descubrimientos de la ciencia contemporánea ha terminado por parecerse a lo natural, semeja su comportamiento. El comportamiento de la naturaleza es impredecible -como las consecuencias de las acciones de los hombres- y esto ha sido descubierto por la ciencia contemporánea.

Según Arendt, aunque los científicos pierden el contacto con el mundo real al intentar descubrir o develar lo que está más allá del mundo sensible, no son ellos los que intervienen en la naturaleza o crean procesos similares a los de la naturaleza. La que hace este trabajo es la técnica, $y$, aunque Arendt reconoce que es esta la que devuelve el contacto de los conocimientos de ciencia con la realidad, con el mundo de los hombres, la técnica interviene creando procesos similares a los de la naturaleza.

Hay dos aspectos relevantes en esta visión: por una parte, la concepción tanto de la historia como de la naturaleza como proceso, es decir como algo que el hombre ha originado; por otro lado, la confianza de que el hombre puede dominar la naturaleza. Frente a esta seguridad propia del pensamiento moderno, es de resaltar, de la mano de Arendt, que lo que el hombre ha hecho al concebir tanto la historia y la naturaleza como proceso es trasladar el carácter de las acciones a estos campos.

Al interferir en la naturaleza tal y como lo ha hecho la técnica, inspirada en los descubrimientos de la ciencia contemporánea, el hombre está iniciando procesos naturales que él mismo no puede predecir -y en muchos de los casos controlar, como lo hemos visto recientemente-.

En cuanto a la historia, concebirla como proceso, implica eliminar de ella todo elemento o hecho particular que no pueda ser enmarcado dentro del espíritu general de una época. Esto es lo que Arendt llama el sentido del que parte y en el que el historiador cree encontrar la objetividad. Una historia así concebida es la consecuencia de lo que Arendt llama la crisis de lo político, pues su visión griega de la política tiene como eje principal la individualidad y, por ende, la pluralidad, condición primaria para que los hombres puedan encontrarse en el espacio público. 
Cuando un hombre actúa inicia una cadena de reacciones que vienen de los otros hombres (ciudadanos) sobre los que ha recaído la acción inicial. Sin importar que se conozca el inicio de una acción, sus consecuencias y reacciones dentro de la comunidad son impredecibles. De la misma forma actúa la naturaleza, pues Arendt, haciendo referencia a los descubrimientos de la física contemporánea, y particularmente a las afirmaciones de Heisenberg sobre el principio de incertidumbre afirma que "podrían aplicarse tipos distintos de leyes naturales, sin contradicciones, a un mismo hecho físico" (Arendt, 1996b, p. 290). Hoy sabemos que toda investigación científica no está sustentada en un solo punto de vista, por el contrario, pueden existir tantas hipótesis como observadores haya.

En la Antigüedad Arendt cree encontrar un impulso común entre la naturaleza y la historia -la primera por su carácter inmortal y la segunda por su pretensión de hacer inmortales los más fútiles asuntos humanos-. En la época que Arendt observa también encuentra un carácter común entre la visón de la naturaleza y de la historia, este carácter es el de proceso impuesto por el pensamiento moderno. La historia, así comprendida, no es más que el reflejo de crisis política contemporánea, que se ve reflejada en la pérdida del interés por lo singular, por las acciones que posibilitan el espacio político. En torno a la naturaleza, entendida como proceso desde la visión de la ciencia contemporánea y manipulada como un artefacto más del imperio del homo faber a través de la técnica, ha desencadenado consecuencias que amenazan la existencia misma del hombre.

Dicho panorama, visto desde la perspectiva de esta pensadora política, puede incitar a rescatar el espacio político en el cual los hombres logren encontrarse despojados de los intereses del imperio del homo faber y de la competencia por domeñar la naturaleza. 


\section{Referencias}

Arendt, H. (1996a). El concepto de historia antiguo y moderno. En Entre el pasado y el futuro: ocho ejercicios sobre la reflexión política (A. Poljak Zorzut, trad.). Barcelona: Ediciones Península.

Arendt, H. (1996b). La conquista del espacio y la estatura del hombre. En Entre el pasado y el futuro: ocho ejercicios sobre la reflexión política (A. Poljak Zorzut, trad.). Barcelona: Ediciones Península.

Arendt, H. (2005). La condición humana (R. Gil Novales, trad.). Barcelona: Ediciones Paidós.

Romeo, M. (2002). El siglo XX: historiografía e historia. Valencia: Universidad de Valencia. 